\title{
Tumor Lysis Syndrome : Authors' Reply
}

\author{
Aruna Rajendran • Deepak Bansal • \\ Sunit C. Singhi
}

Received: 17 April 2013 / Accepted: 17 April 2013 / Published online: 22 May 2013

(C) Dr. K C Chaudhuri Foundation 2013

To the Editor: We thank Gupta et al. for their interest in our article [1] and bringing up practical queries in the management of tumor lysis syndrome (TLS).

Choice of fluid for hyper-hydration: Five percent dextrose in $1 / 4$ normal saline is the recommended fluid [2]. We have observed iatrogenically induced hyperglycemia as a result of hyper-hydration with fluids containing dextrose. Blood sugar should be monitored; we have managed hyperglycemia by alternating fluid containing dextrose with fluid lacking it.

Relevance of Lactate Dehydrogenase level (LDH): LDH level is a surrogate for tumor proliferation. The higher the level, the greater the risk of TLS [3].

Duration of alkalinization: Alkalinization should be discontinued when: 1) Uric acid normalizes, 2) Cytotoxic therapy begins, 3) Urine $\mathrm{pH}$ exceeds 7.5 , or 4) Hyperphosphatemia develops [2-4].

Grading the severity of TLS: Several attempts have been made to classify and grade TLS; a popular one is the CairoBishop grading classification [5]. As per this grading, laboratory TLS is defined as either a $25 \%$ change or level above or below normal, for any two or more serum values of uric acid, potassium, phosphate, and calcium within $3 \mathrm{~d}$ before or $7 \mathrm{~d}$ after the initiation of chemotherapy. Clinical TLS is defined as the presence of laboratory TLS and any one or more of: 1) Creatinine: $\geq 1.5$ upper limit of normal, 2) Cardiac arrhythmia/sudden death, or 3) Seizure.

\section{References}

1. Rajendran A, Bansal D, Marwaha RK, Singhi SC. Tumor lysis syndrome. Indian J Pediatr. 2013;80:50-4.

2. Fisher MJ, Rheingold SR. Oncologic emergencies. In: Pizzo PA, Poplack DG, eds. Principles and Practice of Pediatric Oncology. 6th ed. Philadelphia: Williams \& Wilkins; 2011. pp. 1143-5.

3. Howard SC, Jones DP, Pui CH. The tumor lysis syndrome. N Engl J Med. 2011;364:1844-54.

4. Ten Harkel AD, Kist-Van Holthe JE, Van Weel M, Van der Vorst MM. Alkalinization and the tumor lysis syndrome. Med Pediatr Oncol. 1998;31:27-8.

5. Cairo MS, Bishop M. Tumour lysis syndrome: New therapeutic strategies and classification. Br J Haematol. 2004;127:3-11.
A. Rajendran $\cdot$ D. Bansal $\cdot$ S. C. Singhi $(\bowtie)$

Department of Pediatrics, Advanced Pediatric Center, Post

Graduate Institute of Medical Education and Research,

Chandigarh 160012, India

e-mail: sunit.singhi@gmail.com 